\title{
Reconnection in NIMROD: Model, Predictions, Remedies
}

T. K. Fowler, R. H. Bulmer, B. I. Cohen, D. D. Hua

June 25, 2003

U.S. Department of Energy

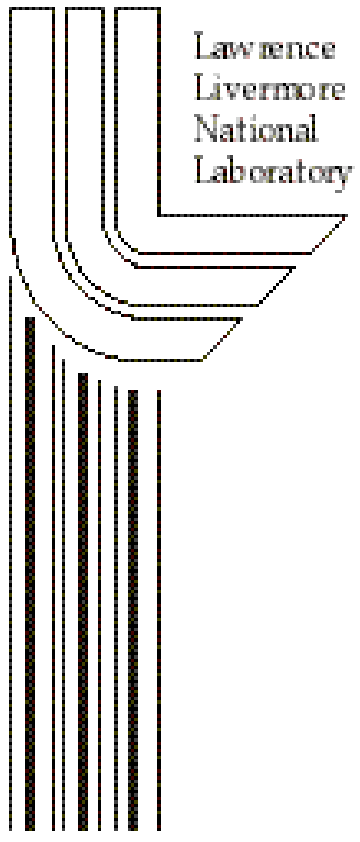


This document was prepared as an account of work sponsored by an agency of the United States Government. Neither the United States Government nor the University of California nor any of their employees, makes any warranty, express or implied, or assumes any legal liability or responsibility for the accuracy, completeness, or usefulness of any information, apparatus, product, or process disclosed, or represents that its use would not infringe privately owned rights. Reference herein to any specific commercial product, process, or service by trade name, trademark, manufacturer, or otherwise, does not necessarily constitute or imply its endorsement, recommendation, or favoring by the United States Government or the University of California. The views and opinions of authors expressed herein do not necessarily state or reflect those of the United States Government or the University of California, and shall not be used for advertising or product endorsement purposes. 
Reconnection in NIMROD: Model, Predictions, Remedies

T. K. Fowler, R. H. Bulmer, B. I. Cohen and D. D. Hua

June 25, 2003

\begin{abstract}
It is shown that in NIMROD the formation of closed current configurations, occurring only after the voltage is turned off, is due to the faster resistive decay of nonsymmetric modes compared to the symmetric projection of the 3D steady state achieved by gun injection. Implementing Spitzer resistivity is required to make a definitive comparison with experiment, using two experimental signatures of the model discussed in the paper. If there are serious disagreements, it is suggested that a phenomenological hyper-resistivity be added to the $\mathrm{n}=0$ component of Ohm's law, similar to hyper-resistive Corsica models that appear to fit experiments. Hyper-resistivity might capture physics at small scale missed by NIMROD. Encouraging results would motivate coupling NIMROD to SPICE with edge physics inspired by UEDGE, as a tool for experimental data analysis.
\end{abstract}

\title{
1. NIMROD Results
}

We consider an example of pulsed injection, Run NIM.3-034, in pillbox geometry with constant resistivity. After the field builds up (to about 1T), the voltage is shut off (crowbar). At that time all lines are open. During decay, lines close within a radius a(t) roughly centered on the symmetric magnetic axis, as shown in Figures 1 - 2 at sequential intervals $\Delta \mathrm{t}$ after crowbar. The symmetric flux surfaces, shown in Figure 3, change little with time. Table 1 gives the approximate closure radius and the $\mathrm{n}=0$ and $\mathrm{n}=1$ magnetic energies $\left(\mathrm{E}_{1,2}\right)$ and the $\mathrm{n}=0$ kinetic energy $\left(\mathrm{K}_{\mathrm{o}}\right)$ and total $\mathrm{K}$ at various intervals $\Delta \mathrm{t}$. Complete histories are plotted in Figure 4.

\begin{tabular}{llllll}
\multicolumn{7}{l}{ Table 1. NIMROD Decay History } \\
$\Delta \mathrm{t}(\mathrm{ms})$ & $\mathrm{E}_{\mathrm{o}}(\mathrm{MJ})$ & $\mathrm{E}_{1}(\mathrm{MJ})$ & $\mathrm{K}_{\mathrm{o}}(\mathrm{J})$ & $\mathrm{K}(\mathrm{J})$ & $\mathrm{a}(\mathrm{t}) /(\mathrm{R} / 2)$ \\
0 & 2.1 & 0.1 & 300 & 375 & open \\
5.6 & 1.8 & 0.09 & 2.3 & 3.4 & open \\
14.4 & 1.6 & 0.08 & 1.75 & 2.7 & $\approx 0.5$ \\
28.9 & 1.3 & 0.05 & 1.5 & 2 & $\approx 0.5$
\end{tabular}


The magnetic energies $\mathrm{E}_{\mathrm{o}}$ and $\mathrm{E}_{1}$ decay approximately exponentially, with time constants:

$$
\begin{array}{llll}
\tau_{\mathrm{o}} & = & 60.3 \mathrm{~ms} & \mathrm{n}=0 \text { decay } \\
\tau_{1} & = & 41.7 \mathrm{~ms} & \mathrm{n}=1 \text { decay }
\end{array}
$$

The symmetric state decay time $\tau_{\mathrm{o}}$ is to be compared with the decay time for the lowest Taylor state in a closed flux conserver (no bias), given by:

$$
\tau=\left(2 \eta \lambda_{\mathrm{o}}{ }^{2} / \mu_{\mathrm{o}}\right)^{-1}=80 \mathrm{~ms}
$$

where $\eta=0.25 \mu_{\mathrm{o}}$ and $\mathrm{R}=1 \mathrm{~m}$ (lowest $\left.\lambda_{\mathrm{o}}=5\right)$.

That $\mathrm{E}_{\mathrm{o}}$ and $\mathrm{E}_{1}$ decay exponentially indicates that they are no longer coupled by instability in decay. We can see this using the spectral formulation of incompressible resistive MHD, in which the system can be described by symmetric and perturbation fields $\mathrm{B}_{\mathrm{o}}$ and $\mathrm{B}_{1}$, the perturbed velocity $\mathrm{v}_{1}$, and the driven flux core field $\mathrm{B}_{\mathrm{FC}}$, giving:

$$
\begin{array}{lllll}
\mathrm{d}\left(1 / 2 \mathrm{~B}_{\mathrm{FC}}{ }^{2}\right) / \mathrm{dt} & = & \mathrm{S} \mathrm{B}_{\mathrm{FC}} & -\mathrm{M}_{\mathrm{FC}} \mathrm{v}_{1} \mathrm{~B}_{1} \mathrm{~B}_{\mathrm{FC}}-\left(\mathrm{B}_{\mathrm{FC}}{ }^{2} / 2 \tau_{\mathrm{FC}}\right)(2) \\
& & \rightarrow & \downarrow \\
\mathrm{dE}_{1} / \mathrm{dt} & = & -\mathrm{M}_{\mathrm{o}} \mathrm{v}_{1} \mathrm{~B}_{1} \mathrm{~B}_{\mathrm{o}}+\mathrm{M}_{\mathrm{FC}} \mathrm{v}_{1} \mathrm{~B}_{1} \mathrm{~B}_{\mathrm{FC}}-\mathrm{E}_{1} / \tau_{1} \\
& & \downarrow & \\
\mathrm{d}\left(1 / 2 \mathrm{~B}_{\mathrm{o}}{ }^{2}\right) / \mathrm{dt} & = & \mathrm{M}_{\mathrm{o}} \mathrm{v}_{1} \mathrm{~B}_{1} \mathrm{~B}_{\mathrm{o}} &
\end{array}
$$

with $\mathrm{E}_{1}=1 / 2\left(\rho \mathrm{v}_{1}{ }^{2}+\mathrm{B}_{1}{ }^{2} / \mu_{\mathrm{o}}\right)$. The steady state existing at $\Delta \mathrm{t}=0$ is given crudely by:

$$
\begin{array}{llll}
\rho \mathrm{v}_{1}{ }^{2}+\mathrm{B}_{1}{ }^{2} / \mu_{\mathrm{o}} & =\mathrm{f} \mathrm{B}_{\mathrm{o}}^{2} / \mu_{\mathrm{o}} & & \text { free energy } \\
\mathrm{v}_{1} \mathrm{~B}_{1} & = & \left(1 / \mathrm{M}_{\mathrm{o}} \lambda_{\mathrm{o}} \tau_{\mathrm{o}}\right) \mathrm{B}_{\mathrm{o}} & \text { from Eq. }(4)
\end{array}
$$

There are two solutions. The one with the smaller value of $v_{1}=\left(1 / M_{0} \lambda_{o} \tau_{o} V_{f}\right) \approx$ $\left(1 / M_{o} S \sqrt{ } f\right) v_{\text {ALFVEN }}$ corresponds to that given by NIMROD $\left(K<<E_{1}\right)$.

Here arrows indicate the direction of energy flow in the driven state. The velocity $v_{1}$ plays a crucial role, whereby the source $S$ (voltage) drives the dynamo $\mathrm{v}_{1} \mathrm{~B}_{1}$ by Eq. (2), which drives the velocity by Eq. (3), which drives $B_{o}$ by Eq. (4). The rapid decay of $v_{1}$ 
$(\propto \sqrt{K})$ after crowbar kills the coupling, giving the observed exponential decay of the magnetic energies at rates like Eq. (1) but with different $\lambda$ 's for different modes.

\section{Effect of Temperature on Decay and Reconnection}

Including ohmic heating and heat transport together with Spitzer resistivity will affect the above results in several ways:

a. When closure occurs, ohmic heating will increase the temperature there, as shown in Figure 5 (for arbitrary $\kappa_{||}=100 \kappa_{\perp}$ ). In future calculations with Spitzer resistivity, this should cause the closed flux to decay more slowly than the open field lines. Closure occurs by the decay of $B_{1} / B_{o}$ (since $B_{o}$ alone is closed). With constant $\eta$, only small differences in $\lambda_{\mathrm{o}}{ }^{2}$ and $\lambda_{1}{ }^{2}$ cause this ratio to decrease, while averaging a temperature-dependent $\eta$ over these states may relatively enhance the decay of $\mathrm{B}_{1}$.

b. Faster decay on the open lines, and on outer surfaces of the closed flux, will cause the $\lambda$ profile to sag (observed in experiments), which may trigger MHD instability during decay (also sometimes observed). In the absence of instability, the only process attempting to maintain the $\lambda$ profile is induction due to decay of the field. Induction due to slow decay of the interior toroidal field cannot sustain the rapidly decaying peripheral poloidal current - including the gun current on open field lines.

c. Delayed closure will delay any rise in temperature and allow the rapid decay of the field everywhere. Closure slow compared to the open line decay time ( $\tau$ in Eq. (1) with temperature for open lines) will lead to the same result, since most of the energy would be lost during closure.

\section{Model}

These observations suggest a model of pulsed injection in NIMROD, described by the following hypotheses:

a. During buildup field lines are open. Instability during buildup is essentially ideal MHD, which preserves open field lines, giving unit current amplification.

b. MHD mode growth saturates in a stable quasi-steady state, probably with a $\lambda$ profile peaked at the geometric axis, with a slope near a stability threshold.

c. Closure happens during resistive decay, not by instability but by the faster decay of non-symmetric modes relative to the symmetrically averaged state, which has closed surfaces. These non-symmetric modes include sheet currents generated during unstable 
growth to preserve open lines. (For tearing modes, the sheets have finite width and instability and closure can occur simultaneously, both at resistive rates.)

$\mathrm{d}$. Stable decay is caused by a window of stability, between the marginal profile peaked on the geometric axis achieved by injection, and an oppositely sloped profile peaked at the magnetic axis. However, with Spitzer resistivity, the $\lambda$ profile should begin to "sag" at the outer edge, since induction cannot sustain the profile at the outer edge, as noted previously. Eventually the sagging $\lambda$ profile may trigger instability again, which could be observed in NIMROD.

\section{Predictions}

The above model provides a qualitative description of NIMROD that can be quantified by introducing the correct anisotropic heat transport and Spitzer resistivity. The model can be used to calculate the useful period for a pulsed reactor between closure and ultimate instability (if this occurs). Multipulse injection could also be calculated, to determine optimum timing allowing closure during decay but avoiding instability in decay.

Comparison of NIMROD results with experiment will shed light on the greatest uncertainty in the model -- the mechanism of closure. The main experimental signature of closure is a rise in temperature (more precisely, pressure) on closed surfaces, to a level determined by the beta that can be achieved by ohmic heating with ion classical or Rechester-Rosenbluth heat transport. A rise in temperature earlier than or higher than that predicted by NIMROD would be a clear signal that non MHD mechanisms cause closure (or at least modes not accessible by NIMROD).

The other important feature of the above model is the fact that the system becomes stable during saturation and decay, allowing a decoupling of the decaying spheromak and decaying gun current. There are two experimental signatures of this prediction. One would be a field decay time that increases as the core temperature increases -- one of the hallmark observations in CTX. The other would be a decay of the gun current faster than the field. To quantify the latter, the maximum gun current that could be sustained inductively by a stable decaying spheromak would be a small fraction of that in steady state, $\mathrm{I}_{\mathrm{GUN}}(0)$, of the order of the ratio of decay times for the open-line gun current and the spheromak, given by:

$$
\mathrm{I}_{\mathrm{GUN}}=(3 / 10)\left(\mathrm{V} / \mathrm{R}_{\Omega}\right) \approx(3 / 10)\left(\mathrm{T}_{\mathrm{E}} / \mathrm{T}\right) \mathrm{I}_{\mathrm{GUN}}(0)
$$

where $\mathrm{V}$ is the loop voltage due to the slowly decaying spheromak toroidal current $\mathrm{I}_{\mathrm{TOR}}$ reduced by $3 / 10$ to account for the sheath; $R_{\Omega}$ is the open line resistance excluding the sheath; $\mathrm{T}_{\mathrm{E}}$ is the open line temperature; and $\mathrm{T}$ is the spheromak core temperature. 
The changes in equilibrium as the gun current decays away are illustrated by Corsica results in Figures 7 and 8, for the bias configuration shown in Figure 6. In Figure $7, \lambda$ is flat, being the same inside and outside the separatrix, giving a gun current comparable to the toroidal current. In Figure $8, \lambda$ is zero outside the separatrix giving zero gun current, rising to a maximum at the magnetic axis inside the separatrix (sagging profile). Note that the location of the spheromak inside the flux conserver is little changed when the gun current goes away -- a fact that persists to very small confined toroidal current relative to a fixed bias. The equilibrium at zero gun current is characterized by zero toroidal field at the separatrix and a poloidal field there matched to that produced by the bias flux compressed in the flux core.

\section{Gun Efficiency}

Aside from sheath loss and other external effects during injection that are not included in NIMROD pillbox calculations, instability occurring in NIMROD (probably ideal MHD) is very efficient at converting injected energy into the symmetric component -about $95 \%$ going into $\mathrm{E}_{\mathrm{o}}$ (mostly the closed spheromak) at crowbar in Table 1. This would be the internal gun efficiency except for energy lost during closure.

The last data point $\Delta \mathrm{t}=28.9 \mathrm{~ms} \approx 0.5 \tau$, at which time $67 \%$ of the $\mathrm{E}_{\mathrm{o}}$ energy remains and the closure radius is about half the spheromak minor radius. How long full closure will take in NIMROD remains to be seen.

An estimate of the importance of fast closure compared to $\tau$ (for open lines) is illustrated by the following model describing decay of the magnetic energy on open lines as the radius of closure a(t) grows in time:

$$
\mathrm{dB}^{2} / \mathrm{dt} \quad=\quad-\quad\left(\mathrm{B}^{2} / \tau\right)\left\{1 \quad-\quad(\mathrm{a}(\mathrm{t}) /(\mathrm{R} / 2))^{2}\right\}
$$

In principle a(t) could be obtained from NIMROD, by fitting data like that given in Table 1 . Lacking sufficient data to obtain a good fit, here we illustrate the point assuming $a(t) /(R / 2)=$ $\left[1-\exp \left(-\mathrm{t} / \tau_{\mathrm{C}}\right)\right]$ with closure time $\tau_{\mathrm{C}}$, giving:

$$
\mathrm{B}^{2}(\mathrm{t}) / \mathrm{B}^{2}(0) \quad \rightarrow \quad \exp \left\{-3 / 2\left(\tau_{\mathrm{C}} / \tau\right)\right\} \quad \text { for } \mathrm{t}>>\tau_{\mathrm{C}}
$$

Thus most of the energy would be lost during closure unless $\tau_{\mathrm{C}}<\tau$ on open lines.

It will also matter when closure occurs. This is illustrated in Figure 9, showing SPHERE calculations approximating a single pulse injection by the SSPX Formation Bank. 
In these calculations delayed closure is approximated by holding the temperature fixed at a low value up to a delay time, after which the usual SPHERE heat transport calculation begins. Four cases are shown, Case 1 with no delay and three other cases with progressively longer delays. Temperatures are in $\mathrm{KeV}$, with $\mathrm{B}$ in tesla. Evidently prolonging ohmic loss at a low temperature during the buildup limits the field and hence the ultimate temperature achieved.

Finally, it matters whether NIMROD correctly calculated saturated MHD amplitudes. Earlier benchmarks of MHD codes found that including compressibility significantly affected MHD amplitudes.

\section{Hyper-resistivity}

If NIMROD results turn out to be inconsistent with experiment, it may be useful to implement into the symmetric Ohm's law a hyper-resistivity already shown to agree with experiment, of the form:

$$
\mathrm{E}_{1 \mid} \quad=\quad \eta \mathrm{j}_{\|}-\nabla \cdot \Lambda \nabla \lambda_{\|}
$$

The hyper-resistive correction should be added only to the $n=0$ component of the Ohm's law as it appears in Faraday's law describing the time evolution of the magnetic field. Implementation would be similar to the hyper-resistive model in Corsica [1] except that in NIMROD it should be applied all the way to the wall including the open-line flux core. Then in effect NIMROD calculates helicity transport by the "dough-hook" (or some symmetric instability) while $\Lambda$ represents processes not in NIMROD.

Here, hyper-resistivity is intended to represent small-scale turbulence not accessible in NIMROD, such as closure effects at the ion Larmor radius scale, or pressure-driven tearing, followed by Rutherford resistive island growth over larger dimensions. We model this by:

$$
\Lambda=\mathrm{C}\left(\mathrm{T}_{\mathrm{E}} / \mathrm{T}\right)
$$

where $\mathrm{C}$ is a fitting constant scaled to the core temperature $\mathrm{T}$ at the magnetic axis calculated by NIMROD and $\mathrm{T}_{\mathrm{E}}$ is the temperature at the outer "edge" (at $\mathrm{r}=0$ at the midplane). Aside from the rapid small scale closure process, this model is similar to "enhanced resistance" discussed in Ref. [2] and it is consistent with the observation that empirical fits of this kind to sustainment shots have succeeded fairly well, with the proviso that $\Lambda$ is smaller at higher temperature [1]. 
The issue is whether hyper-resistivity significantly affects MHD modes that open the flux surfaces. With the model in Eq. (11), it could do so only through changes in the $\lambda$ profile, in competition with MHD control of the profile. At the expected level based on results from Ref. [1], with $\Lambda$ proportional to an appropriately-averaged resistivity, hyperresistivity would have little effect prior to MHD saturation. However, it could aid closure during weak sustainment, by reducing the decay of the symmetric state, first during the late stages of buildup as modes saturate. The latter effect might also benefit closure in pulsed experiments like the Formation Bank. In the decay phase of a pulsed reactor, outward helicity propagation by hyper-resistivity might actually be harmful, by prolonging the coupling of the decaying spheromak to the gun current, but also helpful, by delaying the onset of MHD instability due to sagging $\lambda$.

There may be a logical inconsistency in assuming that short scale processes can give rise to resistive Rutherford island growth in an otherwise MHD stable regime (perhaps implying many close-spaced resonances missed by MHD). Note also that Eq. (11) differs from another ad hoc hyper-resistivity model in Ref. [3] in which $\Lambda$ is proportional to nonsymmetric MHD mode amplitudes, as a model of Rechester-Rosenbluth (R-R) momentum transport in a static tangled field produced by MHD. Even so, significant effects occurred only if $\Lambda$ was larger than expected values based on R-R transport.

We thank Carl Sovinec for his continuing support of our NIMROD simulation activity. This work was performed under the auspices of the U.S. Department of Energy by the University of California Lawrence Livermore National Laboratory under contract no. W-7405-ENG-48.

\section{References}

[1] B. W. Stallard, S. Woodruff, R. H. Bulmer, D. N. Hill, E. B. Hooper, H. S. McLean, R. D. Wood, and the SSPX Team, "Magnetic Helicity Balance in the SSPX Spheromak," Physics of Plasmas, in press.

[2] T. K. Fowler, D. D. Hua and B. W. Stallard, "Spehromak Impedance and Current Amplification,” UCRL-ID-147087, January 31, 2002.

[3] D. D. Hua, T. K. Fowler and E. C. Morse, J. Plasma Phys. 4, 275 (2001). 
Figure 1. NIMROD magnetic field line surface-of-section plot at $\Delta \mathrm{t}=14.4 \mathrm{~ms}$ after crowbar (nim3.034).

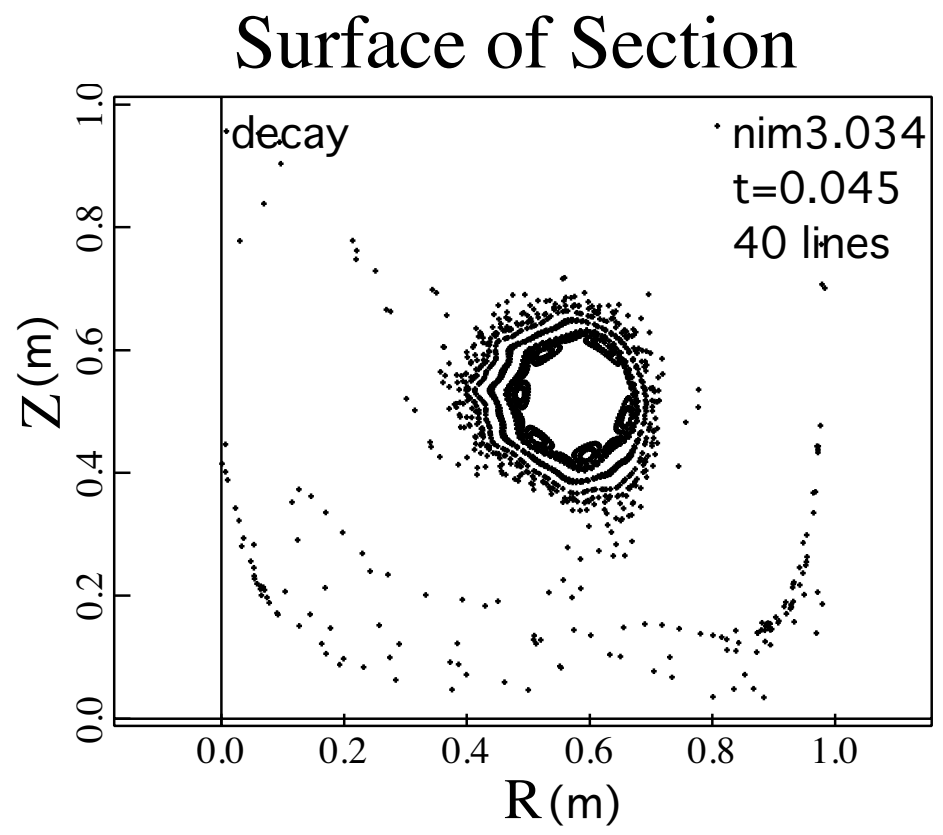

Figure2. NIMROD magnetic field line surface-of-section plot at $\Delta \mathrm{t}=28.9 \mathrm{~ms}$ after crowbar (nim3.034).

Surface of Section

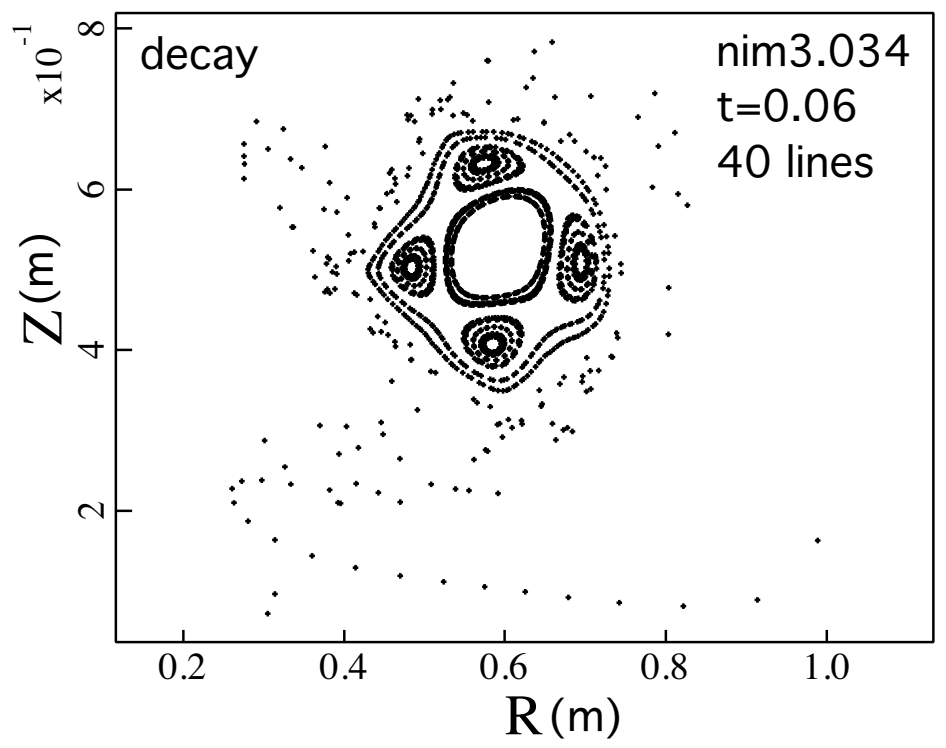


Figure 3. NIMROD toroidally averaged poloidal flux at crowbar of electrostatic drive (nim3.034).

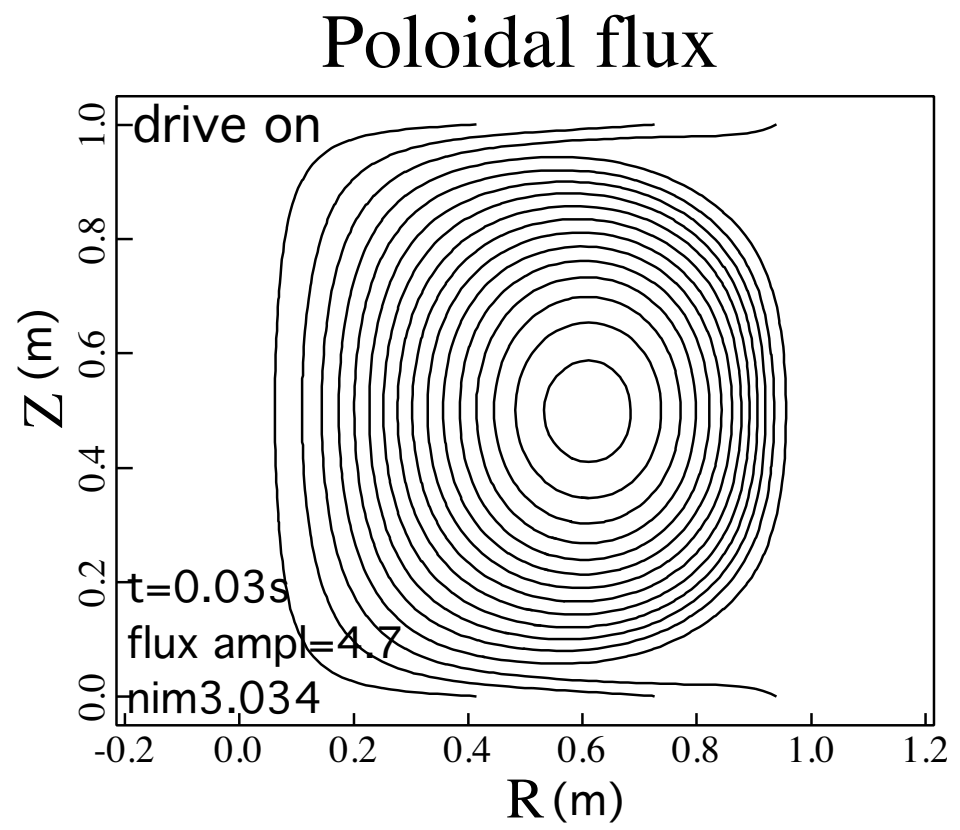

Figure 4. NIMROD magnetic energy time history during decay, resolved by toroidal Fourier harmonic (nim3.034).

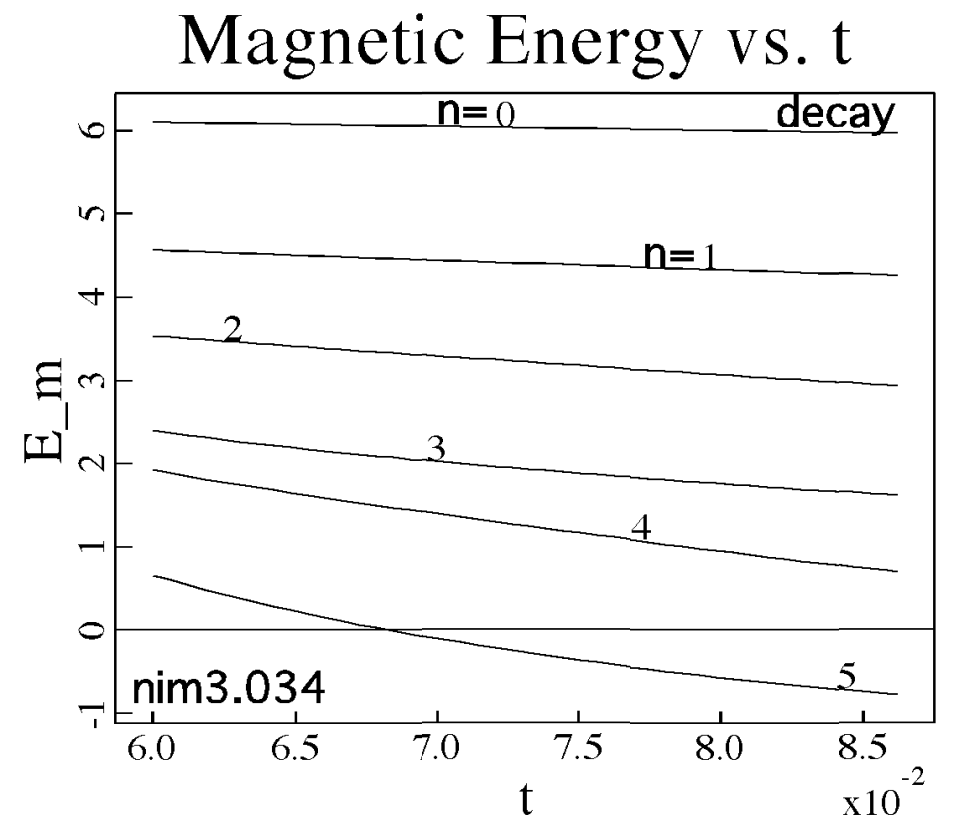


Figure 5. NIMROD simulation of decay showing peaked temperature on closed field lines (nim3.034).

Temperature vs. Radius

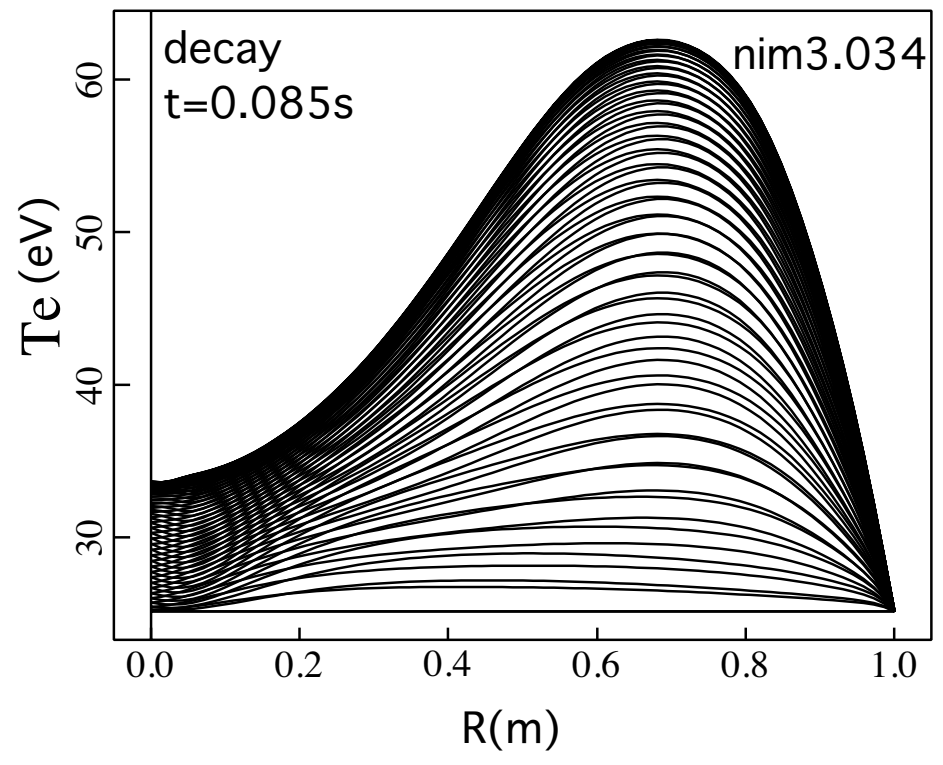


Figure 6. CORSICA plot of bias flux for SSPX vacuum field.

\begin{tabular}{ccc}
\multicolumn{4}{c}{ Bias FluX } \\
Coil currents \\
$\begin{array}{ccc}\text { A } & \text { I/Iref } \\
1 & 0 & 0.00 \\
2 & 0 & 0.00 \\
3 & 0 & -0.00 \\
4 & 0 & -0.00 \\
5 & 0 & 0.00 \\
6 & 0 & 0.00 \\
7 & 0 & 0.00 \\
8 & 0 & -0.00 \\
9 & 800 & 1.00\end{array}$
\end{tabular}

SSPX SOL vacuum field

90.800

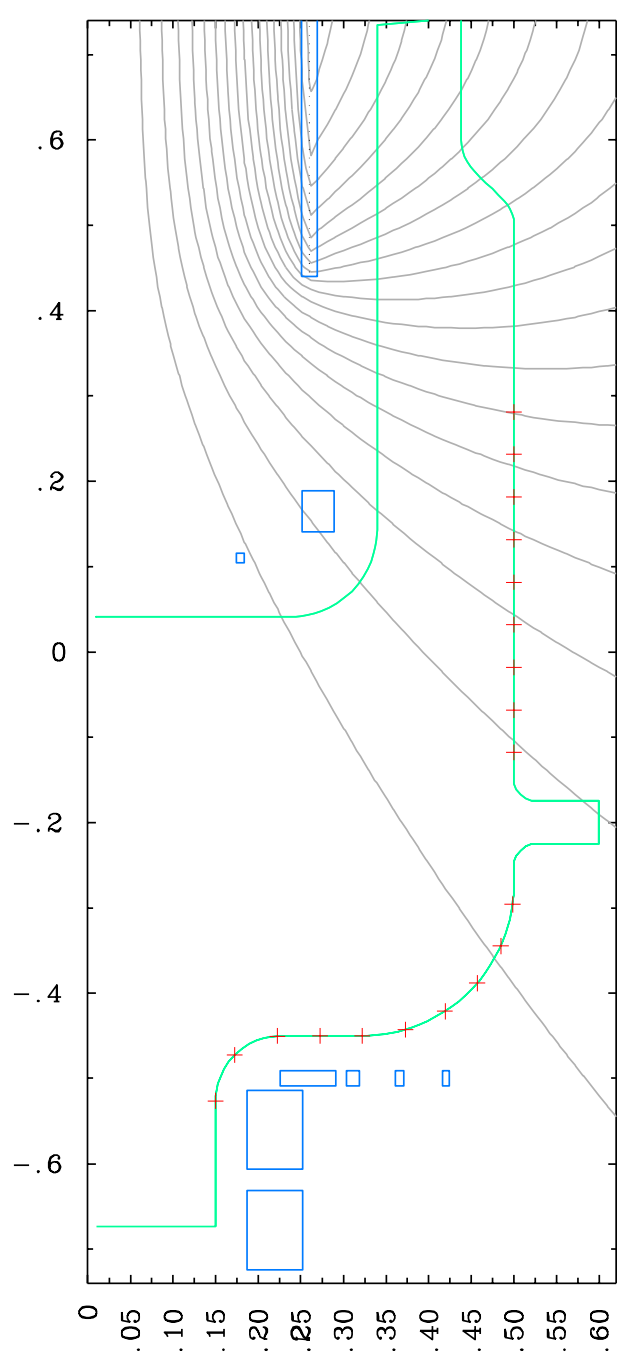

$\Delta \Psi=2.0 \mathrm{mWb}$

$\Psi_{\max }=37.0$

世gun $=33.5$ 
Figure 7. CORSICA plot of SSPX equilibrium with $\mathrm{I}_{\text {gun }}=257 \mathrm{kA}$.

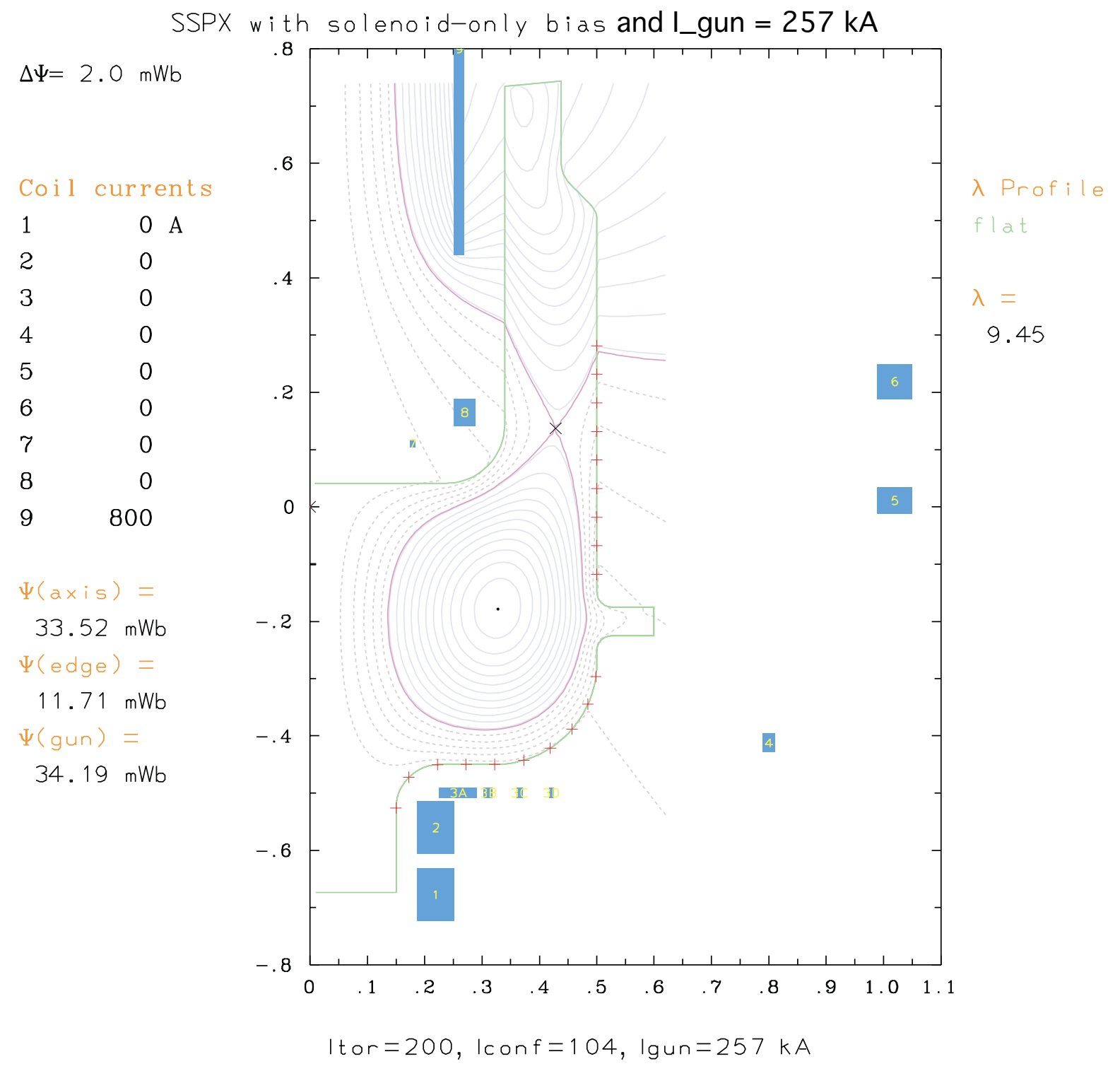


Figure 8. CORSICA plot of SSPX equilibrium with $\mathrm{I}_{\text {gun }}=0$.

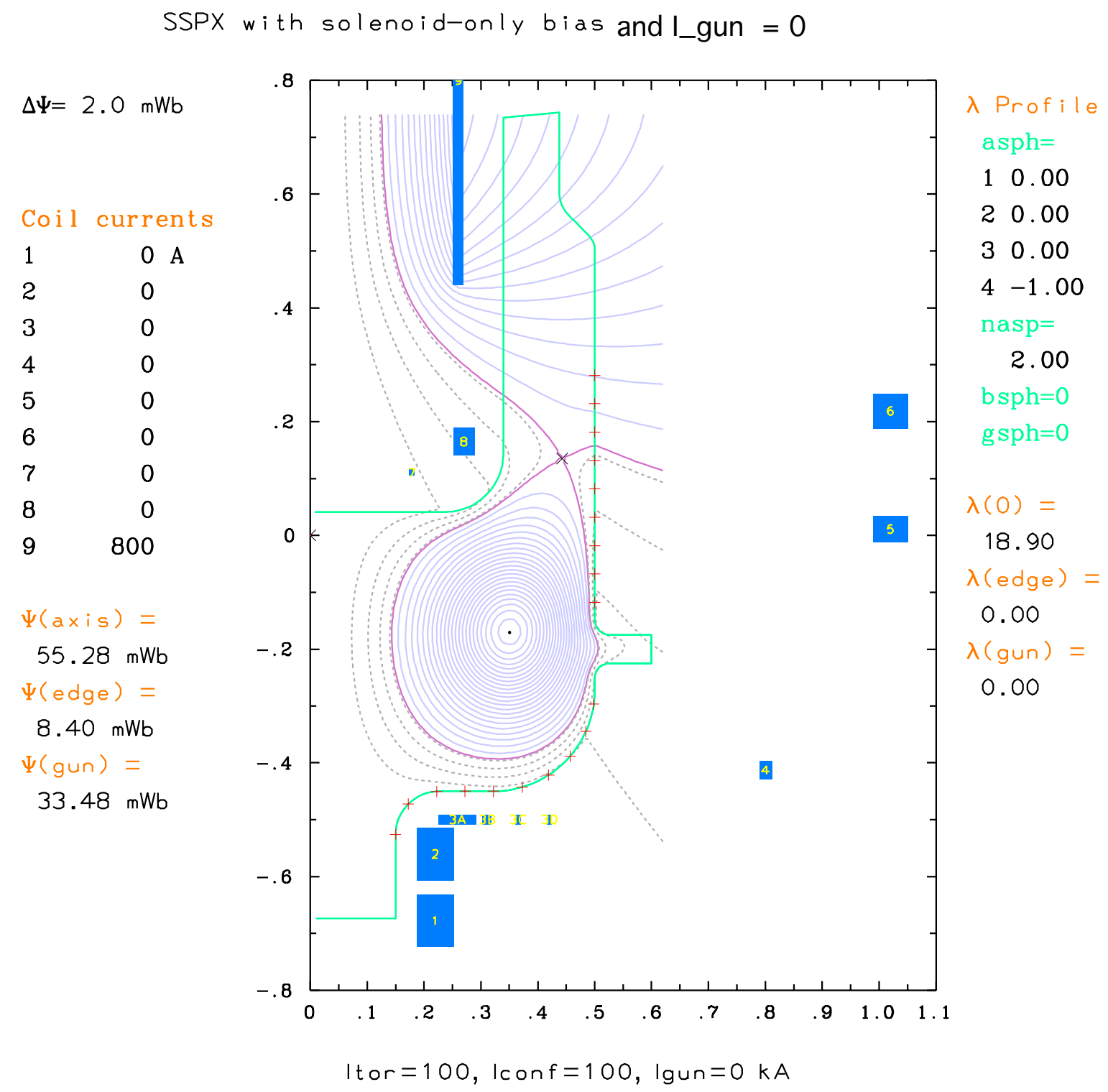


Figure 9. SPHERE calculations of delayed closure.

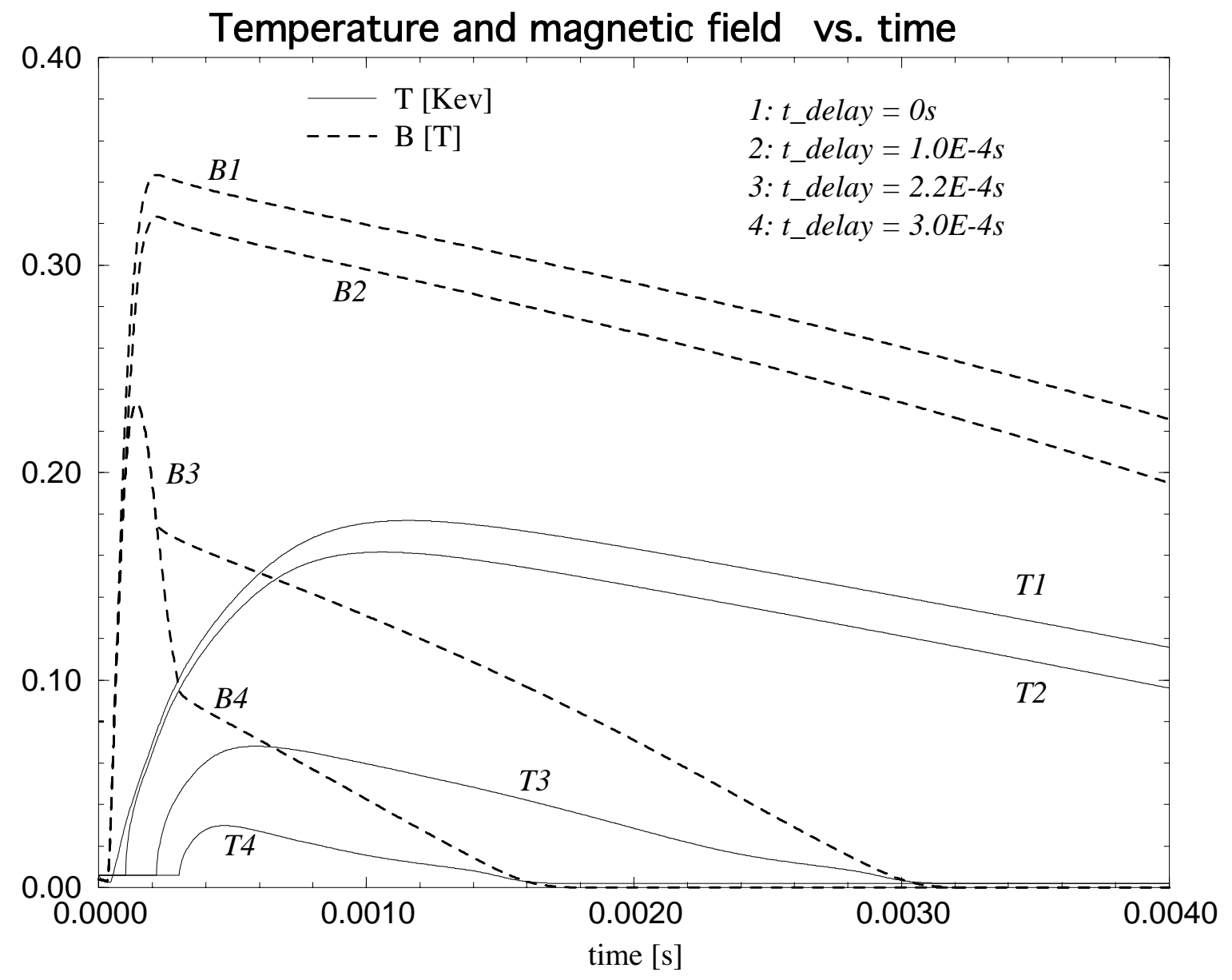

